\title{
Institution-Wide Blood Management Protocol Reduces Transfusion Rates Following Spine Surgery
}

\author{
ALLYSON R. ALFONSO, BS, BA, LORRAINE HUTZLER, MPA, CLAUDETTE LAJAM, MD, \\ JOSEPH BOSCO, MD, JEFFREY GOLDSTEIN, MD \\ NYU Langone Orthopedic Hospital, New York, New York
}

\begin{abstract}
Background: Spine surgery is associated with significant intraoperative blood loss, often leading to transfusion. Patients who receive transfusions have an increased length of stay and risk of perioperative complications. To decrease the transfusion rate, we implemented an evidence-based institution-wide restrictive transfusion blood management guideline. The goal of this study is to describe the impact of this guideline on our spine surgery patients.

Methods: We analyzed the incidence of transfusion following 3709 single-institution, inpatient spine procedures before and after implementation of a revised blood transfusion protocol. The baseline period (1742 patients) from January 2014 to March 2015 was compared to the study period (1967 patients) of April 2015 to July 2016. One patient was excluded because of incomplete medical records. The revised protocol included establishing a postoperative blood transfusion trigger at hemoglobin $<7 \mathrm{~g} / \mathrm{dL}$, instituting a computerized provider order entry, and appointing a physician champion to monitor and report progress.

Results: Transfusion rate decreased from $16.2 \%$ to $9.7 \%$ from baseline to study period, respectively $(P<.001)$. The number of transfusions in patients with hemoglobin $>7 \mathrm{~g} / \mathrm{dL}$ decreased to $4.9 \%$ from $6.1 \%(P=.09)$. The rate of transfusions with a prior hemoglobin test increased from $42.0 \%$ to $59.1 \%(P<.001)$. Length of stay was reduced from 3.67 to 3.46 days $(P=.04)$, and postsurgical infection rate was reduced from $1.5 \%$ to $0.6 \%(P=.01)$. There was no significant difference in total hospital costs following protocol implementation.

Conclusions: Implementation of a restrictive transfusion protocol through use of a computerized provider order entry and a physician champion to oversee clinician compliance led to a $40.1 \%$ reduction in blood transfusion following spine surgery. Behavior changes were visible with a $40.7 \%$ increase in hemoglobin documentation before transfusion, and patients benefited from a reduction in length of stay and postsurgical infection rate. Future study is encouraged to understand the long-term impact of this intervention and its role in hospital expenditure.
\end{abstract}

Research Article

Keywords: transfusion, spine, computerized provider order entry, hospital cost, infection

\section{INTRODUCTION}

Spine surgery is associated with significant intraoperative blood loss, often resulting in transfusion. Willner et $\mathrm{al}^{1}$ completed a systematic review that assessed both pharmacologic and nonpharmacologic factors used to reduce intraoperative blood loss and subsequent transfusion. It has been shown that blood transfusions prolong hospital length of stay, increase postoperative complications, and increase 30-day return to the operating room. ${ }^{2}$ In an activity-based costing model it was estimated that direct and indirect overhead costs were between $\$ 522$ and $\$ 1183$ (mean $\$ 761 \pm \$ 294$ ) per unit of red blood cells transfused. Blood costs have been found to be geographically variable, though these costs have been identified as a source of further study to reduce health care costs. ${ }^{3}$
Restrictive blood transfusion, accomplished by setting a higher threshold to initiate transfusion, has been compared to liberal blood transfusion. Restrictive blood transfusion has been shown to be at least as effective as liberal blood transfusion in critical care $^{4}$ and hip surgery. 5,6 This includes studies with long-term follow-up that showed no significant difference in mortality from cardiovascular disease, cancer, or infections between transfusion groups. ${ }^{6}$ Given comparable safety and outcomes, protocols have been implemented to reduce liberal blood transfusions. Clinician decision support along with targeted education has been effective at reducing liberal red blood cell transfusions at an academic medical center. ${ }^{7}$ This includes computerized provider order entry (CPOE), whereby clinicians must select an indication before blood is dispensed. 
The purpose of this study was to assess the impact of an evidence-based ${ }^{4-7}$ institution-wide restrictive transfusion blood management guideline to reduce the rate of blood transfusions following spine surgery. We predict that implementation of a restrictive transfusion blood management protocol will decrease transfusion rate, length of stay, postsurgical infection rate, and total hospital cost without increasing other complications. Further, guidelines will promote behavior modification assessed by orders for hemoglobin levels prior to transfusion.

\section{MATERIALS AND METHODS}

We analyzed the incidence of red blood cell transfusion in all inpatient spine procedures at a tertiary-care urban teaching hospital before and after implementation of a revised blood transfusion protocol. The revised blood transfusion protocol included establishing a postoperative blood transfusion trigger at hemoglobin $<7 \mathrm{~g} / \mathrm{dL}$ for nonsymptomatic patients and instituting a $\mathrm{CPOE}$ that required clinicians to select an indication for transfusion orders. A physician champion was appointed to educate colleagues, monitor, and report progress.

The baseline period from January 2014 to March 2015 was compared to the study period of April 2015 to July 2016. The primary outcome was transfusion rate following implementation of the restrictive transfusion protocol. Secondary outcome measures included length of stay, total hospital cost, hemoglobin orders prior to transfusion to study protocol compliance, and rate of postsurgical infections, particularly surgical site infections. We also calculated transfusion cost based on Shander et $\mathrm{al}^{3}{ }^{3}$ who estimated that a unit of red blood cells costs between $\$ 533$ and $\$ 1183$. We used the more conservative estimate of $\$ 522$ per unit to calculate cost savings based on transfusion rate.

\section{Statistical Methods}

Statistical analysis was performed using IBM SPSS for Windows, version 23.0 (SPSS, Inc, Chicago, Illinois); $\chi^{2}$ tests were performed for categorical variables and independent samples $t$ tests for continuous variables.

\section{RESULTS}

A total of 3709 inpatient spine procedures were recorded in the baseline (1742 patients) and study
Table 1. Baseline and study period group breakdown.

\begin{tabular}{lcc}
\hline & Baseline & Study Period \\
\hline Mean age \pm SD) & $53.7 \pm 17.1$ & $54.8 \pm 17.5$ \\
Total number of patients & 1742 & 1967 \\
Total number of patients transfused & 283 & 190 \\
Total number of units transfused & 707 & 466 \\
\hline
\end{tabular}

(1967 patients) periods. During the baseline period, 283 patients received transfusions during the baseline period, and 190 patients received transfusions during the study period. One patient was excluded because of incomplete medical records. There was no significant difference in patient age between groups $(P=.051)$ as shown in Table 1 .

Figure 1 shows the transfusion rate decreased from $16.2 \%$ baseline to $9.7 \%$ in the study period $(P<.001)$. Secondary outcomes are shown in Table 2. The number of transfusions in patients with a hemoglobin $>7 \mathrm{~g} / \mathrm{dL}$ decreased to $4.9 \%$ from $6.1 \%$ $(P=.09)$. The rate of transfusions with a prior hemoglobin test increased from $42.0 \%$ baseline to $59.1 \%$ in the study period $(P<.001)$. The length of stay in the hospital was reduced from 3.67 baseline to 3.46 days during the study period $(P=.04)$. The postsurgical infection rate was also significantly reduced from $1.5 \%$ to $0.6 \%$ from baseline to study period $(P=.01)$. All were surgical site infections, with the exception of 1 patient admitted for signs of sepsis.

There was no significant difference in actual total hospital costs following protocol implementation. Based on the number of units transfused and the estimated cost of a unit of packed red blood cells,

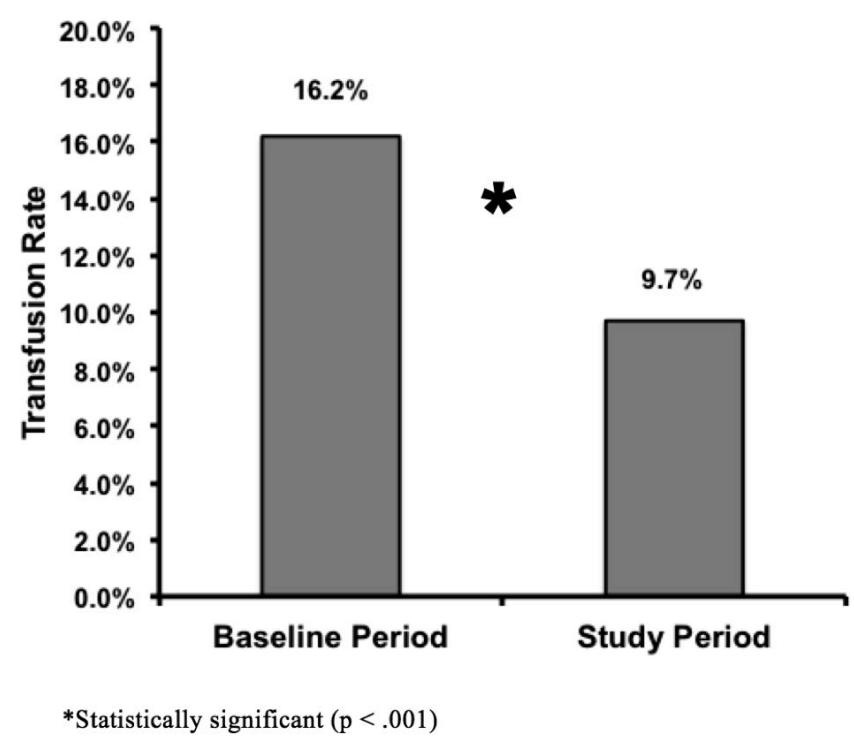

Figure 1. Reduction in transfusion rate. 
Table 2. Reduction in length of stay and postsurgical infections, increase in hemoglobin testing, and equivocal mean total cost.

\begin{tabular}{|c|c|c|c|}
\hline & Baseline & Study Period & $P$ Value \\
\hline Length of stay (mean days $\pm \mathrm{SD}$ ) & $3.67 \pm 3.20$ & $3.46 \pm 2.93$ & .04 \\
\hline Rate of transfusions with a prior hemoglobin test (\%) & 42.0 & 59.1 & $<.001$ \\
\hline Postsurgical infection (\%) & 1.5 & 0.6 & .01 \\
\hline Mean total hospital cost per patient (outcome value \pm SD) & $\$ 184776.53 \pm \$ 131478.49$ & $\$ 192673.83 \pm \$ 136152.80$ & .07 \\
\hline Total cost of transfusions ${ }^{\mathrm{a}}$ & $\$ 369054.00$ & $\$ 243252.00$ & - \\
\hline
\end{tabular}

${ }^{\mathrm{a}}$ Based on estimate by Shander et $\mathrm{al}^{3}$ of average cost of $\$ 522$ per unit of packed red blood cells.

the cost savings from decrease in transfusion was $\$ 125802$. This is a $34.1 \%$ reduction in cost of packed red blood cells from the baseline period.

\section{DISCUSSION}

Our study demonstrates that an institution-wide restrictive transfusion blood management guideline with CPOE and an appointed physician champion can reduce the blood transfusion rate, instigate behavioral change, and reduce both length of stay and the postsurgical infection rate. This singleinstitution restrictive transfusion protocol reduced the blood transfusion rate following spine surgery by $40.1 \%$. Behavior change was observed with a $40.7 \%$ increase in hemoglobin test ordering and documentation before transfusion, and patients benefited from a reduction of length of stay in hospital. This is consistent with previous results reporting reduction in liberal red blood cell use and reduced length of hospital stay following implementation of the CPOE system. ${ }^{7}$

We found postsurgical infection significantly decreased from $1.5 \%$ to $0.6 \%$ from baseline to study period $(P=.01)$. This association between health care-associated infection and red blood cell transfusion has been previously reported. A retrospective cohort study of 3721 patients following lumbar spine surgery found an increased risk of surgical site infection and overall postoperative infection after exposure to allogeneic blood transfusion. ${ }^{8}$ These results are supported by 2 preceding retrospective case-control studies that found blood transfusion to be a risk factor for surgical site infections $s^{9,10}$ and, moreover, volume of blood transfusion was found to play a role. ${ }^{10}$ In a systematic review and meta-analysis of randomized clinical trials with restrictive versus liberal red blood cell transfusion strategies, it was shown that a restrictive transfusion protocol decreased the incidence of serious health care-associated wound infection, though not infection overall. ${ }^{11}$ In the present study, postsurgical infections were all surgical site infections, with the exception of 1 patient who presented with signs of sepsis. Though several studies have reported reduction in the infection rate with restrictive transfusion protocol, the Centers for Disease Control and Prevention guideline for the prevention of surgical site infection currently does not recommend withholding transfusions for the sole purpose of preventing surgical site infections. $^{12}$

Contrary to our prediction, there was no significant difference between the baseline and study period mean total hospital cost. Potential confounding factors, which should be further studied, include implementation of International Classification of Diseases, Tenth Revision (ICD-10) in October $2015^{13}$ during the study period and/or other costly institution-wide program implementation, including inpatient telemetry. Trentino et $\mathrm{al}^{14}{ }^{14}$ in a retrospective cohort study, took into account confounders of hospital cost in transfusion such as hospital type, patient age, sex, admission type, discharge type, adjusted diagnosis-related group, and comorbidities. They found that the mean cost was 1.83 times higher for inpatients transfused than for those not transfused. ${ }^{14}$ In this study, looking particularly at blood transfusion cost, it was estimated that $\$ 125802$ was saved by reducing the number of transfused units. This is a conservative estimate as we used the lower of the estimated costs per unit published by Shander et al. ${ }^{3}$ It has also been found that blood costs are often underestimated and vary geographically. ${ }^{3}$ Outside of orthopedic surgery, the number of units of transfused blood received intraoperatively in liver transplantation was found to be the strongest predictor of total hospital cost and service area costs. This correlated with not only operating room costs, but also intensive care unit, pharmacy, diagnostic, and ancillary costs. ${ }^{15}$ Together, these factors suggest that not only may cost savings be underestimated, but that future research should focus on the reasons for this variation and how cost can be controlled.

Future directions related to the implemented protocol include long-term follow-up to assess the 
sustainability and the impact on the standard of care. Studies assessing restrictive and liberal blood transfusion protocols have investigated the impact of transfusion on health care outcomes, as well as strategies to decrease transfusion requirements. ${ }^{1}$ These methods include preoperative cessation of antiplatelet and anticoagulation medication; intraoperative considerations such as cell salvage, anesthetic management, and use of antifibrinolytic agents (eg, tranexamic acid); surgical technique; and postoperative considerations of surgical drain placement and choice of analgesic. ${ }^{16}$

There are several limitations of this singleinstitution retrospective cohort study. There were no uniform criteria for operative treatment and all spine procedures were included, which may have introduced unidentified confounders to the data set. Each physician champion also had individual methods to provide feedback after the initial educational intervention. This can impact the protocol's reproducibility. To assess proper protocol implementation, this study evaluated the rate of transfusion with a prior hemoglobin test and transfusion rate with a hemoglobin $>7 \mathrm{~g} / \mathrm{dL}$. This did not take into account clinical scenarios in which transfusion may have been warranted contrary to protocol. Additionally, retrospective data collection limited the ability to control for factors affecting hospital cost during the study period, such as implementation of ICD-10 and inpatient telemetry, and patient demographics such as medical comorbidities.

Ultimately the restrictive transfusion protocol studied can be expanded to additional institutions in order to evaluate generalizability of the protocol and maintenance of effects over time. There is a need to further investigate the effect on hospital cost, which includes controlling for confounding factors and identifying additional ways to safely reduce resource use.

\section{CONCLUSION}

Implementation of a restrictive transfusion protocol through use of CPOE and a physician champion to oversee clinician compliance met the primary goal of reducing blood transfusions following spine surgery at a single institution. Secondary outcome measures found improvement in hemoglobin documentation before transfusion and reduced length of hospital stay and rate of postsurgical infections following protocol implementation. Al- though overall hospital costs were not reduced in the study period, in a conservative estimate there was a $34 \%$ reduction in transfusion-specific spending. Future study is encouraged to better understand the long-term impact of this intervention and the role it plays in hospital costs.

\section{REFERENCES}

1. Willner D, Spennati V, Stohl S, Tosti G, Aloisio S, Bilotta F. Spine surgery and blood loss: systematic review of clinical evidence. Anesth Anal. 2016;123(5):1307-1315. https://doi.org/ 10.1213/ANE.0000000000001485

2. Seicean A, Alan N, Seicean S, Neuhauser D, Weil RJ. The effect of blood transfusion on short-term, perioperative outcomes in elective spine surgery. J Clin Neurosci. 2014;21(9):15791585. https://doi.org/10.1016/j.jocn.2014.03.003

3. Shander A, Hofmann A, Ozawa S, Theusinger OM, Gombotz H, Spahn DR. Activity-based costs of blood transfusions in surgical patients at four hospitals. Transfusion. 2010;50(4):753-765. https://doi.org/10.1111/j.1537-2995.2009. 02518.x

4. Hébert PC, Wells G, Blajchman MA, et al. A multicenter, randomized, controlled clinical trial of transfusion requirements in critical care. $N$ Engl J Med. 1999;340(6):409-417. https://doi. org/10.1056/NEJM199902113400601

5. Carson JL, Terrin ML, Noveck H, et al. Liberal or restrictive transfusion in high-risk patients after hip surgery. $N$ Engl J Med. 2011;365(26):2453-2462. https://doi.org/10.1056/ NEJMoa1012452

6. Carson JL, Sieber F, Cook DR, et al. Liberal versus restrictive blood transfusion strategy: 3-year survival and cause of death results from the FOCUS randomised controlled trial. Lancet. 2015;385:9974:1183-1189. https://doi.org/10.1016/S01406736(14)62286-8

7. Saag HS, Lajam CM, Jones S, et al. Reducing liberal red blood cell transfusions at an academic medical center. Transfusion. 2017;57(4):959-964. https://doi.org/10.1111/trf.13967

8. Janssen SJ, Braun Y, Wood KB, Cha TD, Schwab JH. Allogeneic blood transfusions and postoperative infections after lumbar spine surgery. Spine J. 2015;15(5):901-909. https://doi. org/10.1016/j.spinee.2015.02.010

9. Schwarzkopf R, Chung C, Park JJ, Walsh M, Spivak JM, Steiger D. Effects of perioperative blood product use on surgical site infection following thoracic and lumbar spinal surgery. Spine (Phila Pa 1976). 2010;35(3):340-346. https://doi. org/10.1097/BRS.0b013e3181b86eda

10. Woods BI, Rosario BL, Chen A, et al. The association between perioperative allogeneic transfusion volume and postoperative infection in patients following lumbar spine surgery. J Bone Joint Surg Am. 2013;95(23):2105-2110. https://doi.org/10.2106/JBJS.L.00979

11. Rohde JM, Dimcheff DE, Blumberg N, et al. Health careassociated infection after red blood cell transfusion: a systematic review and meta-analysis. JAMA. 2014;311(13):1317-1326. https://doi.org/10.1001/jama.2014.2726

12. Berrios-Torres SI, Umscheid CA, Bratzler DW, et al. Centers for Disease Control and Prevention guideline for the prevention of surgical site infection, 2017. JAMA Surg. 2017;152(8):784-791. https://doi.org/10.1001/jamasurg.2017.0904 
13. Centers for Medicare and Medicaid Services. ICD-10. https://www.cms.gov/medicare/Coding/ICD10/index.html. Accessed December 29, 2016.

14. Trentino KM, Swain SG, Burrows SA, et al. Increased hospital costs associated with red blood cell transfusion. Transfusion. 2015;55(5):1082-1089. https://doi.org/10.1111/trf. 12958

15. Ruiz J, Dugan A, Davenport DL, Gedaly R. Blood transfusion is a critical determinant of resource utilization and total hospital cost in liver transplantation. Clin Transplant. 2018;32(2):e13164. https://doi.org/10.1111/ctr.13164

16. Bible JE, Mirza M, Knaub MA. Blood-loss management in spine surgery. $J$ Am Acad Orthop Surg. 2018;26(2):35-44. https://doi.org/10.5435/JAAOS-D-16-00184

Disclosures and COI: Each author certifies that he or she has no commercial associations (eg, consultancies, stock ownership, equity interest, patent or licensing arrangements, etc.) that might pose a conflict of interest in connection with the submitted article. Completed ICJME disclosures for each author can be viewed at http://www.icmje.org/ coi_disclosure.pdf.

Corresponding Author: Lorraine Hutzler, MPA, NYU Langone Orthopedic Hospital, 301 East 17th St, Suite 1402, New York, NY 10003. Phone: (212) 598-6048; Fax: (212) 598-3883; Email: Lorraine.Hutzler@nyumc.org.

Published 30 June 2019

This manuscript is generously published free of charge by ISASS, the International Society for the Advancement of Spine Surgery. Copyright (C) 2019 ISASS. To see more or order reprints or permissions, see http://ijssurgery.com. 